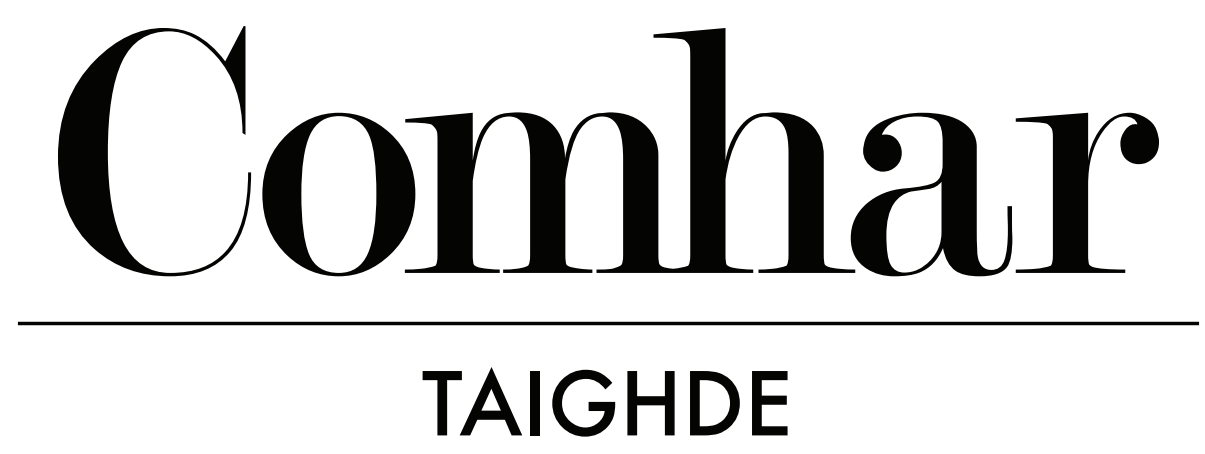

EAGRÁN 6

Samhain 2020

Alt Taighde

Birlinn Chlann Raghnaill: Long a fuair foscadh in Éirinn

Dáta foilsithe:

30 Samhain 2020

Cóipcheart:

(c) Peadar Ó Muircheartaigh, 2020 (CC BY-NC)

Seoladh gréasáin:

https://comhartaighde.ie/eagrain/6/omuircheartaigh/

\author{
Údar: \\ Peadar Ó Muircheartaigh
}

\section{Comhfhreagras:}

Peo5@aber.ac.uk

Seoladh seasmhach (DOI):

https://doi.org/10.18669/ct.2020.10

Is le cabhair deontais i gcomhair tograí Gaeilge a d'íoc an tÚdarás um Ard-Oideachas trí Choláiste na hOllscoile, Corcaigh le tacaíocht Choiste Léann na Gaeilge, Litríocht na Gaeilge agus na gCultúr Ceilteach, Acadamh Ríoga na hÉireann, a fhoilsítear an ríomhiris seo. Aithníonn COMHAR an tacaíocht seo.

ISSN: 2009-8626 


\title{
Birlinn Chlann Raghnaill: Long a fuair foscadh in Éirinn
}

\author{
Peadar Ó Muircheartaigh
}

\section{Achoimre}

Déantar luathstair an dáin Albanaigh Bìrlinn Chlann Raghnaill le hAlasdair Mac Mhaighstir Alasdair a chíoradh san alt seo. Scrúdaítear luathchóip den dán a chaomhnaítear i measc pháipéirí Chathail Uí Chonchúir (1710-1791) in Acadamh Ríoga na hÉireann, d'fhonn léargas a thabhairt ar luathstair théacsúil an dáin. Léirítear gur chuir an scoláire Albanach Seumas MacLathagain (1728-1805) cóip an Acadaimh chuig Cathal Ó Conchúir am éigin tar éis Feabhra na bliana 1771, agus gur bhailigh MacLathagain an téacs le linn turais a thug sé ar Innse Gall i samhradh na bliana 1770. Fágann an fhianaise nua seo gur bailíodh leagan MhicLathagain de Birlinn Chlann Raghnaill sé bliana sular fhoilsigh mac an fhile an dán den chéad uair.

\section{Réamhrá}

'S thug sinn buidheachas don Ard-rìgh chum na dùilean

Deagh Chlann Raghnaill a bhith sàbhailt o bhàs brùideil.

'S an sin bheum sinn a siùil thana, bhallach, thùilinn

'S leag sinn a crainn mhìn-dearg, ghasda fad a h-ùrlair,

'S chuir sinn a-mach ràimh chaol bhasgant dhaite, mhìne

Den ghiuthas a bhuain MacBharrais an Eilean Fhìonain,

'S rinn sinn an t-iomradh rèidh tulganach gun dearmad,

'S ghabh sinn deagh longphort aig barraibh Charraig Fheargais.

Thilg sinn acraichean gu socair anns an ròd sin,
Ghabh sinn biadh is deoch gun airceas's rinn sinn còmhnaidh. (Thomson 1996: 153)

Sin mar a thagann Birlinn Chlann Raghnaill('Clanranald's Galley') agus a foireann i dtír in Éirinn agus mar a thagann an dán fada seo leis an bhfile Alasdair Mac Mhaighstir Alasdair, alias Alasdair MacDhòmhnaill / Alexander MacDonald (c. 1698-c. 1770), go críoch. Tá déantús seo Mhic Mhaighstir Alasdair le háireamh i measc mórdhánta na Gaeilge a cumadh sa dara leath den ochtú céad déag. Ní beag sin le rá agus sinn ag trácht ar na blianta a thug Caoineadh Airt Ui Laoire le hEibhlín Dubh Ní Chonaill, Cúirt an Mheán Oíche le Brian Merriman, agus Moladh Beinn Dòbbrain le Donnchadh Bàn Mac an t-Saoir dúinn. Go deimhin, d'fhéadfaí a áitiú go bhfuil Bìrlinn Chlann Raghnaill chun cinn ar na mórdhánta sin, ó thaobh na sofaisticiúlachta agus na haeistéitice de. Ach oiread leis na dánta clúiteacha eile sin, tá a rian fágtha ag Birlinn Chlann Raghnaill ar litríocht Ghaeilge ár linne, ar an 
dá thaobh de Shruth na Maoile. Ba é a thug inspioráid do Shomhairle MacGill-Eain agus a dhán fada féin, An Cuilthionn, á chumadh aige (Dymock 2009: 163) agus tá a lorg fágtha go láidir ar an úrscéal mara Aois Fir le Liam Ó Muirthile (2015).

Ó tharla go bhfuil ár dtuiscint ar litríocht an dá Ghaeilge - idir litríocht na linne seo agus litríocht an ochtú céad déag - saibhrithe chomh mór sin ag léamha cáiréiseacha Mháire Ní Annracháin, ní miste liom san aiste ghearr seo iniúchadh a dhéanamh ar ghné amháin de stair théacsúil Birlinn Chlann Raghnaill, dán a fháisceann Gaeil Éireann agus Alban lena chéile agus atá ina ábhar inspioráide do scríbhneoirí an dá Ghaeilge go fóill. Díreofar ar chóip lámhscríofa den dán a breacadh san ochtú céad déag agus a chaomhnaítear fós in Acadamh Ríoga na hÉireann, cóip a léiríonn nach naisc mheafaracha amháin a d'fháisc agus a fháisceann Gaeil Éireann agus Alban le chéile ach teagmhálacha leanúnacha na litríochta, an léinn agus an chairdis.

\section{Cúlra Bìrlinn Chlann Raghnaill}

Ainm réasúnta nua is ea Birlinn Chlann Raghnaill a tháinig chun cinn sa naoú céad déag (Thomson 1996: 153), ach is i lár an ochtú céad déag a chum Alasdair Mac Mhaighstir Alasdair, duine de phríomhfhilí na Gaeilge Albanaí, an dán is cáiliúla dá chuid. Tá an chosúlacht ar an scéal gur lean próiseas na cumadóireachta ar feadh i bhfad: cé nach bhfuil cinnteacht ar bith ag baint leis an scéal, deirtear gur tosaíodh ar an obair le linn do Mhac Mhaighstir Alasdair a bheith ina bháille ar Eilean Chanaigh timpeall na bliana 1751 ach gurbh in Uibhist a críochnaíodh é beagnach fiche bliain ina dhiaidh sin (Bateman 2012: 74; Thomson 1996: 134). Cineál ar leith soithigh an 'bhirlinn' (nó 'birling' i nGaeilge na hÉireann, de réir $F G B$ ) atá ina shainchomhartha liteartha agus cultúrtha de chuid Innse Gall agus cósta iarthar na hAlban le fada (Parsons 2013; Bateman 2008; 2012; Gillies 2013a: 80). Tagraíonn 'Clann Raghnaill' i dteideal an dáin do thaoiseach na craoibhe sin de chuid na nDòmhnallach, taoiseach a dtugtar 'Clanranald' nó 'The Captain of Clanranald' air i mBéarla (nó Mac Mhic Ailein sa Ghaeilge). Dream iad Clann Raghnaill a raibh ceannas acu ar Uibhist a Deas, Beinn na Faoghla, Eilean Eige, Eilean Chanaigh agus Àird nam Murchan agus a samhlaíodh, go traidisiúnta, mar 'the backbone of Gaelic culture and Jacobitism in the Highlands' (Campbell 1984: 33).

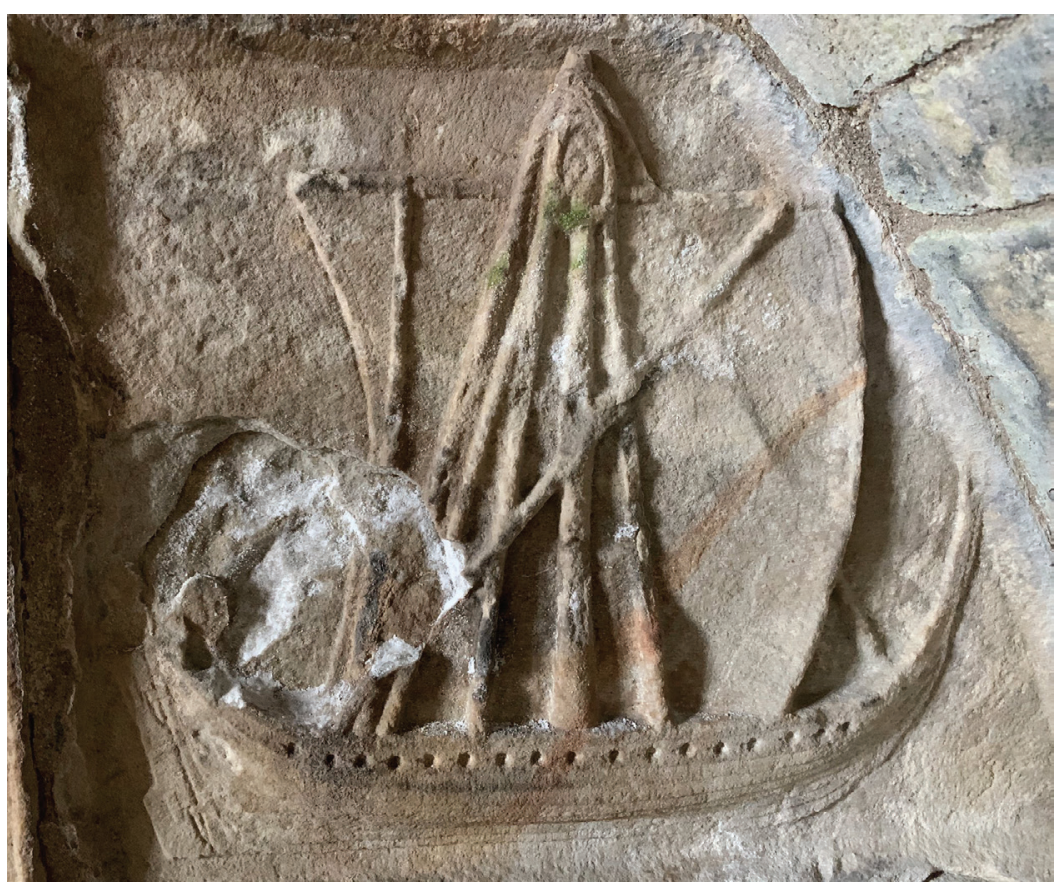

Figiúr 1

Dealbh de bhirling ó thuama Alasdair MhicLeòid, alias Alasdair Crotach (16ú céad) in Tùr Chliamhainn, Roghdal, Na Hearadh. Grdwiele / CC BY-SA 3.0

Déantús eisceachtúil filíochta é Bìrlinn Chlann Raghnaill, a bhfuil 600 líne véarsaíochta ann, nach mór, a bhfuil giotaí próis fite fuaite leo agus struchtúr sofaisticiúil meadarachta ina bhunchloch faoin 'prosimetrum' seo (Thomson 1996: 26-27). Murab ionann is cuid de véarsaíocht eile a linne a bhraith ar an seachadadh béil, cuid de véarsaíocht Mhic Mhaighstir Alasdair san áireamh, is cosúil nár mhair Birlinn Chlann Raghnaill riamh ach mar phíosa a léití amach os ard (Campbell 1961: 39-40; cf. Gillies 2013b: 212). Cur síos atá sa dán, ó thús deireadh, ar aistear mara, ar gach dár bhain don bhirling loinge agus dá foireann ó ullmhaíodh an bád agus ó cuireadh chun farraige í in Uibhist Innse Gall ar Lá Bríde go dtí gur tháinig sí i dtír i gCarraig Fheargusa ar chósta Aontroma, mar a raibh foscadh, 'deagh longphort' agus 'biadh is deoch gun airceas' ar fáil. Idir an dá linn, ríomhann an file mar a théann foireann na loinge $i$ 
ngleic le stoirm mhór agus le huafáis na mara. Tarraingíonn tús an dáin ar phaidreacha Sheoin Carswell um bheannú loinge a fhaightear in Foirm na n-Urrnuidheadh, an chéad leabhar Gaeilge a cuireadh i gcló i nDún Éideann sa bhliain 1567. I gcodanna eile den dán, cloistear macallaí chóraithe catha théacsanna na Meán-Ghaeilge agus na Nua-Ghaeilge Moiche, Cath Fionntrágha agus Agallamb na Seanórach ina measc, agus cur síos á dhéanamh ag an bhfile ar an mbirling féin agus ar fhoireann na loinge (MacLeod 1933: 118-25; Bateman 2012: 81). Ní iontas, dá réir sin, é go ndéanann Dòmhnall Meek cur síos ar Mhac Mhaighstir Alasdair mar 'the heir of the classical literary tradition' (Meek 1999: 260).

Dá fhréamhaithe sa traidisiún Gaelach é Bìrlinn Chlann Raghnaill mar dhán, áfach, is déantús filíochta de chineál nua ar fad é, faoi mar atá áitithe ag Raghnall MacIlleDhuibh (Black 2006), Meg Bateman (2012: 81) agus Somhairle MacGill-Eain féin (1985: 34). Eipic is ea é a eascraíonn ní hamháin ó thraidisiúin na litríochta Gaeilge ach ó chultúr agus smaointeoireacht na hAlban in aimsir na hEagnaíochta (Black 2006: 119) agus a bhfuil fealsúnacht Sheacaibíteach an fhile lárnach ann (Pittock 2012). Dar le Ruairidh MacThòmais, an duine is déanaí a thug faoi chuid de shaothar Mhic Mhaighstir Alasdair a chur in eagar, is é an dán seo, thar déantús ar bith eile dá chuid, 'the ultimate demonstration of Mac Mhaighstir Alasdair's hard, exact intellectual power' (Thomson 1990: 173).

\section{Cóip Acadamh Ríoga na hÉireann}

Tá cóip Acadamh Ríoga na hÉireann de Bìrlinn Chlann Raghnaill ceangailte anois ina cuid den lámhscríbhinn ARÉ LS E ii 1. Meascán mearaí de bhileoga fánacha a bhí i seilbh Chathail Óig Uí Chonchúir (1710-91), fear Bhéal Átha na gCarr, tráth, atá inti (Murphy agus Wulff 1936: 2287-2301). De shliocht seanríthe Chonnacht é Ó Conchúir, scoláire cáiliúil Gaeilge an ochtú céad déag. Fuair sé oiliúint mhaith i léann traidisiúnta na Gaeilge ó sheanscoláirí thuaisceart Chonnacht, baill de theaghlaigh léannta a bhí ag freastal ar uaisle an cheantair leis na céadta bliain roimhe sin (Ó Catháin 2015). Bhí an-cháil ar Ó Conchúir, in Éirinn agus thar lear, go mór mór as a shaineolas i dtaobh chúrsaí staire agus luathfhoinsí Gaeilge na staire. Léargas atá sa lámhscríbhinn iom- lán ar réimsí spéise Uí Chonchúir i gcaitheamh a shaoil agus cúrsaí staire, giotaí as na ginealaigh agus dánta a bhain le muintir Uí Chonchúir go mór chun cinn inti.

Seo mar a rinne garmhac Uí Chonchúir (An tAth. Charles O'Conor) cur síos ar an gcuid seo de lámhscríbhinn a sheanathar sular tháinig sí go dtí an tAcadamh Ríoga:

An Irish Poem, entitled "Beannachadh Luinge, the blessing of a Ship.”- It is in the Irish language, in English hand-writing, and is a modern transcript, quoted by Martin in his Western Islands. In this copy the verses are about 340; they are in that idiom of the Irish, which is corruptly called Erse ... (O'Conor 1818: 171).

Bhí dul amú bunúsach ar an Ath. O’Conor sa mhéid is nach é an dán seo atá ag Martin Martin ar chor ar bith ach leagan den phaidir a d'fhoilsigh Carswell sa bhliain 1567, paidir a bhí coitianta i measc an phobail, más fíor don Mháirtíneach: 'A Form of Prayer us'd by many of the [Hebridean] Islanders at sea after the Sails are hoisted' (Martin 1716: 127). Is léir nár aithin an tAth. O'Conor gur dán de chuid an ochtú céad déag a bhí os a chomhair.

Beagán le cois céad bliain tar éis don Ath. O'Conor í a chlárú den chéad uair, cláraíodh an lámhscríbhinn arís, le linn do lámhscríbhinní eile an Acadaimh a bheith á gclárú. Seo an cuntas a bhaineann leis an gcuid seo den lámhscríbhinn:

125 (108) (Hand Qbegins here [= lámh anaithnid]). Gu'm beannuigheadh Dia long Chlann Raonuil.

$24+36+33+30+5+24+8+6+6+6+10+15$

$+10+7+8+94$ lines of verse, separated by short paragraphs of prose describing the circumstances. Headed "Beannachadh luinge, maille re prosnachadh fairge, rinneadh do sgiob \& do bhirlinn le Tighearna Chlann Raonuill" [i.e., MacDonald of Clan Ronald]. The lower portion of p. 132 where this Scottish Gaelic piece ends, is blank except for the note "Fechain pinn, \&tc" (written by Hand A) (Murphy agus Wulff 1936: 2300).

Is ionann 'Hand A' agus scríbhneoireacht Chathail Uí Chonchúir, rud a dheimhníonn go raibh na bileoga 


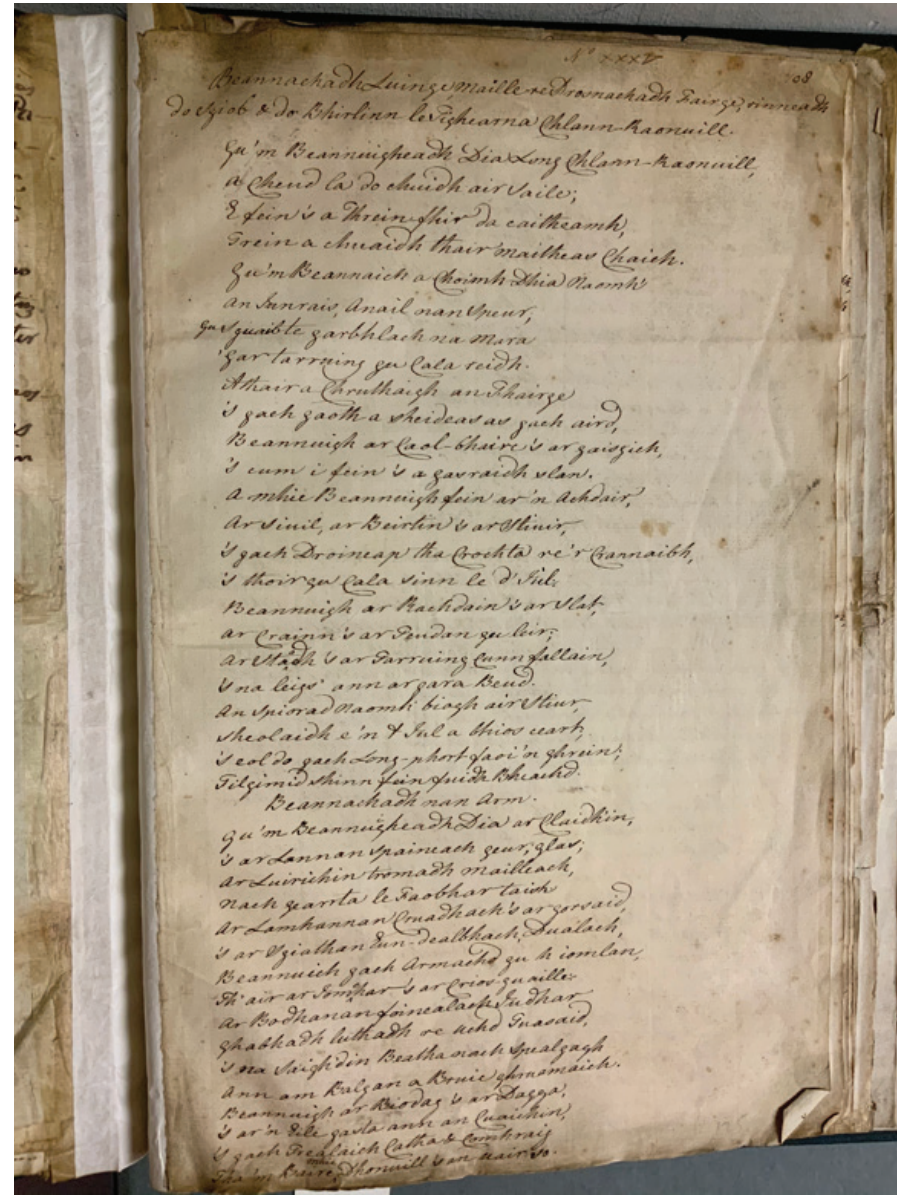

Figiúr 2

ARÉ LS E ii 1, Ich 125. Le caoinchead Leabharlann Acadamh Ríoga na hÉireann $@$

seo ina sheilbh aige tráth éigin lena bheo. Níor aithin lucht cláraithe na lámhscríbhinne an scríobhaí ar bhaist siad 'Hand Q' air, áfach, agus is é an t-aon sampla den scríbhneoireacht sin atá sa lámhscríbhinn. Cuireadh leagan seo an Acadaimh i gcló chomh fada siar le 1961 nuair a d'fhoilsigh an scoláire Iain Latharna Caimbeul (1906-1996) eagrán den téacs (Campbell 1961). Ach oiread le lucht cláraithe na lámhscríbhinne, níor aithin an Caimbeulach lámh a breactha agus níor fhéad sé, dá réir sin, ainm scríobhaí a lua léi. Ní raibh aon eolas ar fáil don Chaimbeulach faoi dháta na lámhscríbhinne ach oiread. Tá tábhacht ar leith leis an gcóip seo i gcomhthéacs stair an dáin.

Tá cuid de na bunphointí téacsúla fós le soiléiriú, agus scagadh den uile chineál fós le déanamh ar an dán (cf. Gillies 2012: 119). Go deimhin, le blianta beaga anuas tá aird ar leith dírithe ar chóip mhistéireach seo Acadamh Ríoga na hÉireann ag Raghnall MacIlleDhuibh (Black 2001) agus ag Uilleam MacGill'Ìosa (Gillies 2012) araon. I ndíolaim thábhachtach d'fhil- íocht na Gaeilge Albanaí ón ochtú céad déag, mar shampla, tuairimíonn MacIlleDhuibh go bhféadfaí go raibh comhfhreagras idir an file agus Cathal Ó Conchúir agus gurbh é an comhfhreagras seo ab údar inspioráide, b'fhéidir, do Mhac Mhaighstir Alasdair (Black 2001: 473). In aiste thábhachtach leis, d'áirigh Uilleam MacGill'İosa 'the implications of the Royal Irish Academy copy found amongst the papers of Charles O'Conor of Belanagare' ar cheann de na buncheisteanna téacsúla sin atá le hiniúchadh go fóill (Gillies 2012: 119). Is é atá fúm a dhéanamh sa chuid eile den aiste ghearr seo roinnt de na himpleachtaí sin a thabhairt i ngrinneas.

\section{Na Foinsí Eile}

Maidir leis na foinsí téacsúla is luaithe dá bhfuil againn, seachas cóip an Acadaimh, níl ach dhá chóip iomlána eile den dán ar marthain. Foinse chlóite is ea ceann díobh sin agus lámhscríbhinn atá sa cheann eile. Foilsíodh an chéad cheann sa díolaim, Comb-chruinneachidh Orainnaigh Gaidhealach (1776), nó Díolaim Eilean Eige ('The Eigg Collection') mar is fearr aithne anois air. Díolaim thoirtiúil filiochta atá thar a bheith tábhachtach i gcomhthéacs fhilíocht Ghaeilge na hAlban é seo. Raghnall MacDhòmhnaill, alias Raghnall Dubh / Ranald MacDonald (c. 1728-1808), mac Alasdair Mhic Mhaighstir Alasdair, a chuir an díolaim i dtoll a chéile agus is ina dhiaidh atá an díolaim ainmnithe ó tharla go raibh Raghnall ina fhear taca (.i. fear ionaid) ag Caiptín Chlann Raghnaill ar Eilean Eige. Tagann cuid mhór dár cuireadh i gcló sa díolaim ó bhailiúchán a athar agus samhlaítear údarás ar leith a bheith ag baint leis an gcóip chlóite de Bìrlinn Chlann Raghnaill dá réir.

Fágtar ansin sinn leis an gcóip lámhscríofa eile den dán. Tá an chóip sin i Leabharlann Ollscoil Ghlaschú, mar chuid de bhailiúchán mór Gaeilge a cuireadh le chéile sa dara leath den ochtú céad déag, a bronnadh ar an leabharlann ag tús na haoise seo caite agus atá cláraithe anois mar GUL MS 1042/15. Ba é an tOirmhinneach Seumas MacLathagain (1728-1805) a chuir an bailiúchán sin i dtoll a chéile. Cainteoir dúchais Gaeilge ab ea MacLathagain a rugadh in oirdheisceart Ghaeltacht na hAlban. Cuireadh oideachas air i gColáiste Chill Rímhinn sular oirníodh ina mhinistir de chuid na hEaglaise Albanaí é. Scoláire, file agus 
foclóirí a bhí in MacLathagain, fear a bhí gafa go hiomlán le hamhráin agus le litríocht na Gaeilge ar feadh a shaoil. Ó lár na 1760idí ar aghaidh, bhí MacLathagain ina shéiplíneach in 'Am Freiceadan Dubh', reisimint Ghaelach de chuid Arm na Breataine agus chaith tréimhsí i Meiriceá, in Éirinn agus ar Oileán Mhanann (Dziennik 2015; Ó Muircheartaigh 2016). Maireann a cháil, go speisialta, mar dhuine de na bailitheoirí litríochta ba bhisiúla in Albain san ochtú céad déag, duine de ghrúpa beag ministrí Protastúnacha (Savonius-Wroth 2019). Tá aird dírithe as an nua

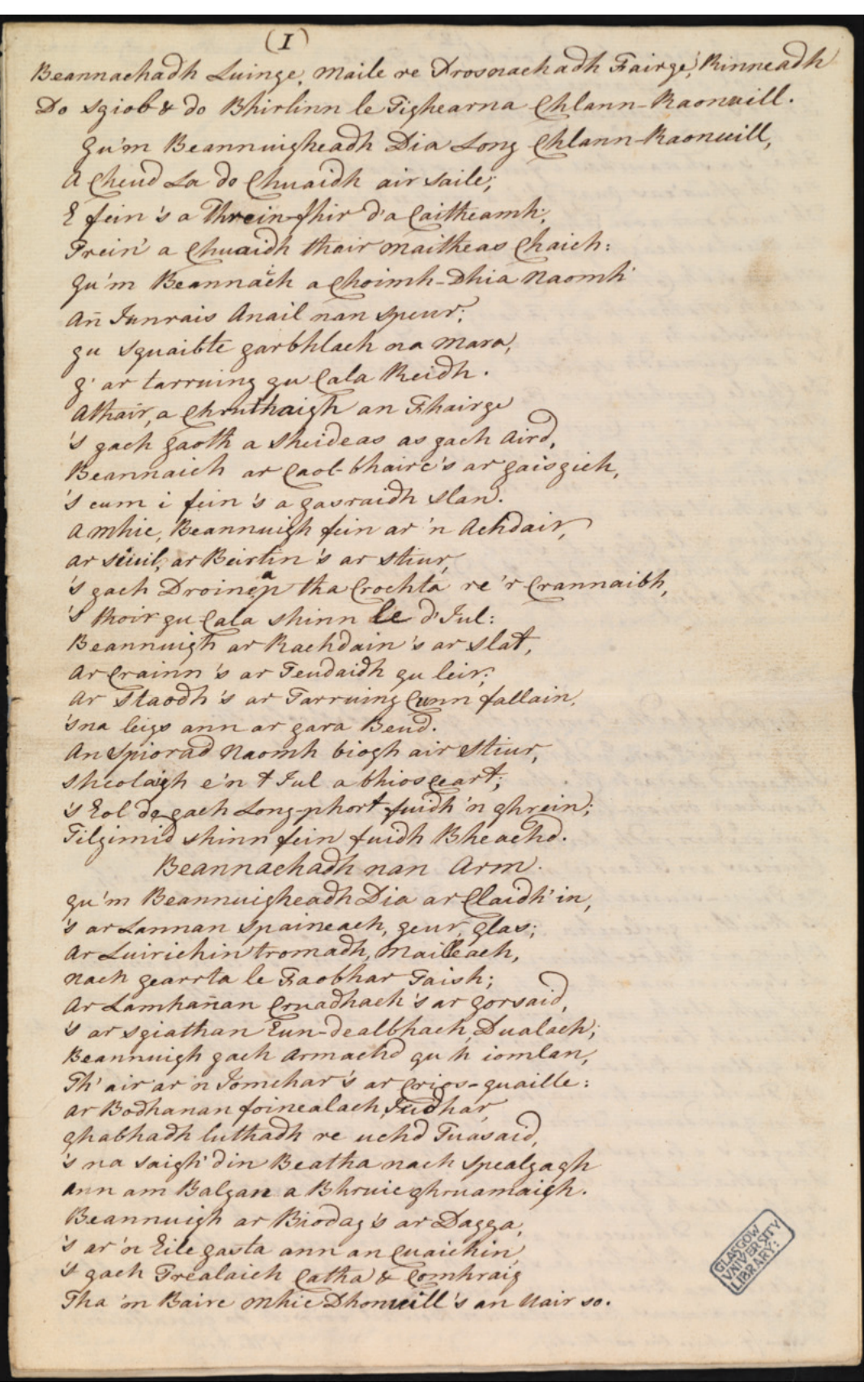

air féin agus ar a bhailiúchán le blianta beaga anuas (Innes 2016; Ó Muircheartaigh 2016).

Bíodh is go raibh lámhscríbhinní MhicLathagain i seilbh Leabharlann Ollscoil Ghlaschú ó bhí 1910 ann, ní raibh aon chlár ar fáil le linn don Chaimbeulach a bheith i mbun eagarthóireachta ar an dán agus níorbh eol dó go raibh luathchóip lámhscríofa eile den dán ar marthain. MacLathagain féin a bhreac an chóip seo atá anois i nGlaschú. In ainneoin gur chuir Ruairidh MacThòmais léamha ó chóip Ghlaschú san áireamh agus an dán á chur in eagar aige, níor foilsíodh cóip Leabharlann Ollscoil Ghlaschú ina hiomláine fós (Thomson 1996: 132-165). D'aithin MacThòmais an dlúthchosúlacht théacsúil idir cóip an Acadaimh agus cóip Ghlaschú agus d'aithin na himpleachtaí a bhain leis an gcosúlacht sin:

The McL 15 and RIA texts are extremely similar, though not identical and one must conclude that they are both copies of the same original, or just possibly that one is a copy of the other (Thomson 1996: 133).

Údar iontais, áfach, nár bhac MacThòmais leis an gcosúlacht idir an dá chóip seo a fhiosrú. Ní doiligh an chosúlacht a mhíniú; Seumas MacLathagain a bhreac an dá cheann. Is é sin le rá, is ionann 'Hand Q' lucht cláraithe lámhscríbhinní Acadamh Ríoga na hÉireann agus scríbhneoireacht Sheumuis MhicLathagain.

Tugadh le fios thuas go bhfuil trí cinn de luathfhoinsí téacsúla i gceist le Birlinn Chlann Raghnaill. Is fíor sin i gcónaí ach is léir anois go síolraíonn cóip Ghlaschú agus cóip an Acadaimh araon ón leagan céanna, leagan a bhí i seilbh Sheumais MhicLathagain. Maidir le líon na leaganacha iomlána de, mar sin, níl i gceist ach Leagan MhicLathagain agus Leagan Díolaim Eilean Eige. Agus an tuiscint nua seo againn ar chóip an Acadaimh, ar chóip Ghlaschú agus ar an ngaol atá eatarthu, is

Figiúr 3

GUL MS 1042/15. Le caoinchead Glasgow University Library ${ }^{\circ}$ féidir cróineolaíocht Leagan Eige agus Leagan MhicLathagain a bheachtú de bheagán. Chuige sin, ní mór caidreamh Uí Chonchúir le MacLathagain a scrúdú. 


\section{Seumas MacLathagain agus Cathal Ó Conchúir}

Ar feadh cuid mhaith dá shaol, bhí MacLathagain ina shéiplíneach in 'Am Freiceadan Dubh' nó 'The Black Watch' mar a bhí air i mBéarla, reisimint Ghaelach de chuid Arm na Breataine. Tar éis roinnt mhaith blianta a chaitheamh i Meiriceá, tháinig Am Freiceadan Dubh go hÉirinn sa bhliain 1767 agus an séiplíneach ina dteannta. Tuairiscíodh a dteacht go hÉirinn ar na nuachtáin:

Cork, Aug 24. Yesterday evening arrived at Cove the Liberty transport in 28 days from Philadelphia, with the 42nd or Royal Highland regiment of foot, commanded by Lieut Gen Lord John Murray. This regiment, consisting of two battalions, left Europe in 1756 and 1758, and served in America and the West Indies during the war, in which service they lost above 60 officers and 2000 men (Belfast Nerwsletter, 1 Meán Fómhair 1767).

Bhí faoiseamh éigin le fáil in Éirinn ag na saighdiúirí Albanacha le hais a raibh fágtha ina ndiaidh acu ach bhí an reisimint siúlach go maith le linn a seala in Éirinn. Tar éis dóibh teacht i dtír sa Chóbh, chaith siad tréimhsí éagsúla i nGaillimh, i nDoire, i mBéal Feirste agus i mBaile Átha Cliath (Ó Muircheartaigh 2016). Le linn dó a bheith lonnaithe i mBaile Átha Cliath is ea a chuir MacLathagain aithne ar Chathal Óg Ó Conchúir. Ba nós leis an gConchúrach cuid mhór den bhliain a chaitheamh i mBaile Átha Cliath, áit a raibh teacht aige go héasca ar dhaoine eile a raibh spéis sa léann acu. I measc na ndaoine sin a bhain le 'ciorcal' seo Uí Chonchúir san ardchathair bhí Ardeaspag Bhaile Átha Cliath, an Dr Seán Mac an tSaoir alias John Carpenter (1729-1786), Charles Vallancey (1731-1812), agus an scríobhaí bisiúil Muiris Ó Gormáin (c. 1710-c. 1788). Bhí MacLathagain i mBaile Átha Cliath le linn na mblianta 1769 , 1770 agus arís in 1774 ach is beag tagairt do MhacLathagain i saothar foilsithe Uí Chonchúir. Faightear tagairt amháin sa réamhrá a chuir sé le The Ogygia Vindicated, áit a luann Ó Conchúir a theagmháil le MacLathagain agus é ag trácht ar na comhráite agus díospóireachtaí a bhí acu lena chéile faoi litríocht an dá Ghaeilge: no man [...] will deny that an Highland bard of the last or present century, may not possess as much poetical fire as the song of Fingal, or any other illiterate bard in Fingal's army. I have the greater pleasure in my conviction on this head, as some short Gaelic originals (of North Britain) highly poetical, have been shewn to me here in Dublin by a very worthy and learned gentleman, the Rev. Mr. Mac Lagan, chaplain to the Highland regiment quartered in our garrison till the beginning of last summer [1774] (O'Conor 1775: xv).

Ní fhaightear ach tagairt ghonta amháin do MhacLathagain i litreacha foilsithe Uí Chonchúir; agus é ag scríobh chuig a mhac 23 Márta 1771, gabhann Ó Conchúir buíochas leis as litir le MacLathagain a chur ar aghaidh chuige: 'I thank you for the care you took in forwarding Mr MacLagan's letter from Belfast. He is a worthy gentleman' (Ward, Wrynn agus Coogan Ward 1988: 245). Tá teacht go fóill ar chuid mhór de na litreacha a cuireadh chuig Ó Conchúir. Ceanglaíodh a chuid litreacha le chéile in imleabhar mór a chaomhnaítear sa lá atá inniu ann in Acadamh Ríoga na hÉireann agus a bhfuil an uimhir ARÉ LS B i 2 ag gabháil leis. I measc na litreacha seo a cuireadh chuig Ó Conchúir, tá péire a scríobh MacLathagain sa bhliain 1771 .

Agus é ag scríobh chuig an gConchúrach ó Bhéal Feirste i mí Feabhra na bliana 1771, rinne MacLathagain cur síos ar an tréimhse a chaith sé in Innse Gall an samhradh roimhe sin (.i. 1770). Tar éis dó slán a fhágáil ag an gConchúrach i mBaile Átha Cliath thug MacLathagain faoi chósta iarthar na hAlban. Lios Mòr, oileán beag amach ó chósta an Òbain, ba cheanncheathrú dó le linn an turais, áit a raibh a sheanchara Dòmhnall MacNeacail (1735-1802), bailitheoir bisiúil eile, ina mhinistir. Ar an turas seo is ea a bhailigh MacLathagain cóip de Birlinn Chlann Raghnaill nó an 'Beannughadh Luinge' mar a thugann sé air, faoi mar a mhíníonn sé féin maille le cur síos spéisiúil ar an dán mar 'a Highland giant':

In this journey I got many songs and poems numbers of which are pretty good, and among others the Beannughadh Luinge which I think was mentioned in a letter I show'd you. It is like a Highland giant, all huge bones and sinews 
(MacLathagain chuig Ó Conchúir, 18 Feabhra 1771, ARÉ LS B i 2).

\section{MacLathagain agus Turas $\mathbf{1 7 7 0}$}

Go bhfios dom, thug MacLathagain dhá chuairt, ar a laghad, ar Innse Gall le linn a shaoil. Ceann i samhradh na bliana 1774 a bhfuil tuairisc fhada againn uirthi i gcín lae Bhéarla a scríobh MacLathagain agus a chaomhnaítear i gCartlann na Cathrach, Dùn Deagh (Turner 2018). Thug sé cuairt eile ar Innse Gall cúpla bliain roimhe sin, i samhradh na bliana 1770. Maidir le turas 1770, níl de thuairisc dhíreach againn air ach an dá litir ghearra a chuir MacLathagain chuig Ó Conchúir:

After returning to Lismore ... I set out for Mull and then to I Colm Kill ... In this journey I got many songs and poems numbers of which are pretty good, and among others the Beannughadh Luinge which I think was mentioned in a letter I show'd you. It is like a Highland giant, all huge bones and sinews. I was in Sky, the Uists and Barra. I have only time to tell you that their hospitality is still in its vigor (MacLathagain chuig Ó Conchúir, 18 Feabhra 1771, ARÉ LS B i 2).

Chuir MacLathagain leis an tuairisc sin, de bheagán, ina chéad litir eile chuig Ó Conchúir:

I returned from I to Mull and travelled north to Tobermory a charming harbour ... Thence I sailed to Ardnamorachan, and thence again to Sky by the little Isle of Egg ... (MacLathagain chuig Ó Conchúir, 31 Bealtaine 1771, ARÉ LS B i 2).

Leanann MacLathagain ar aghaidh sa litir chéanna le cur síos ar chósta Uibhist. Faraor, ní thugann sé féin le fios cén áit go díreach ar bailíodh an dán, ach is féidir a thuilleadh fianaise a chur sa mheá chun an scéal a shoiléiriú. Is léir gur ó fhoinse scríofa a bhailigh MacLathagain Bìlinn Chlann Raghnaill, murab ionann agus dánta eile a bhreac sé ó bhéalaithris. Tá roinnt féidearthachtaí láidre ann maidir le log a bhailithe, ach tá an cás a bhaineann le cuid acu níos láidre ná a chéile: Àird nam Murchan agus an ceantar máguaird, Eilean Eige, agus Uibhist a Deas. Codanna de dhúiche Chlann Raghnaill iad na háiteanna sin ar fad, agus áiteanna iad a raibh baint ar leith ag Mac Mhaighstir Alasdair agus a theaghlach leo (Black 1986: 4; Campbell 2000; Stiùbhart 2012).

Glactar leis gur timpeall na bliana 1770 a d'éag Alasdair Mac Mhaighstir Alasdair (Thomson 1996: 13; Black 1986: 5) ach ní hann d'aon tuairisc chomhaimseartha ar a bhás. Ceist a bhaineann le hábhar mar sin — ar casadh MacLathagain agus Mac Mhaighstir Alasdair ar a chéile ar thuras MhicLathagain? Tá sé i seanchas Àrasaig gur ann, ó thuaidh de leithinis Àird nam Murchan, a bhásaigh an file agus gur ann chomh maith a cuireadh é (MacDonald 1996). Ní dhéanann MacLathagain trácht ar bith ar a bheith in Àrasaig ach luann sé Àird nam Murchan go sonrach, bíodh is nach dtugann sé le fios sa litir gur sheas sé ann. Dar ndóigh, bhí cuid mhaith dá shaol caite ag Mac Mhaighstir Alasdair in Àird nam Murchan (Black 1986; Thomson 1996: 5). Dá ndéanfaí a mhaíomh gur ar tír mór, in Àird nam Murchan nó sa cheantar máguaird a fuair MacLathagain an dán ó dhuine dá theaghlach nó ón bhfile féin fiú, chaithfí a admháil gur gann an fhianaise eile a thacódh leis an téis. Shamhlófaí go luafaí an file féin sa litir a chuir MacLathagain chuig Ó Conchúir, ach ní luaitear.

Maidir le hEilean Eige, ní deirtear sa litir ach go ndeachaigh MacLathagain go dtí an tEilean Sgitheanach 'by the little Isle of Egg'. Ní féidir a bheith cinnte go ndeachaigh MacLathagain i dtír ar Eilean Eige fiú, ach bhí mac an fhile ina fhear taca ar an oileán ó 1766 ar a dhéanaí (Campbell 2000: 135; ach cf. Thomson 1996: 13 a deir gur 'not later than 1770' a tháinig Raghnall Dubh go hEilean Eige). An bhféadfaí a áitiú, mar sin, gur bhailigh MacLathagain Birlinn Chlann Raghnaill ó mhac an fhile? Agus é ag trácht ar na difríochtaí idir an dá leagan deir MacThòmais, '[i]mportantly, McL and RIA have a few lines not in Eigg but clearly belonging to the poem' (Thomson 1996: 133). Tá dánta eile de chuid Alasdair Mhic Mhaighstir Alasdair i mbailiúchán MhicLathagain i Leabharlann Ollscoil Ghlaschú agus an bhliain 1770 luaite leo. Ar cheann díobh sin tá an dán dar teideal Smeòrach Chlann Raghnaill. Faightear an dán seo i nDíolaim Eilean Eige ach is suntasach go bhfuil leagan MhicLathagain i bhfad níos faide ná leagan Eige (Thomson 1996: 112). Bailíodh na dánta seo de chuid Alasdair Mhic Mhaighstir Alasdair, dánta a 
bhfuil dlúthbhaint acu le Clann Raghnaill, an samhradh céanna. Is é is dóchúla mar sin, gur bailíodh ón bhfoinse chéanna iad. Tá línte iontu araon nach bhfuil i leaganacha Díolaim Eilean Eige, rud a thabharfadh le fios, b'fhéidir, nach ó Raghnall Dubh a fuair MacLathagain iad. An amhlaidh a bhí Raghnall Dubh i dtaobh le luathdhréachtaí a athar ach gur éirigh le MacLathagain teacht ar leagan ní ba chríochnúla an fhile in áit éigin eile? Ba é a thuairimigh Iain Latharna Caimbeul it may well be that the [Royal Irish Academy] text [of Birlinn Chlann Raghnaill] goes back to a fair copy of the poem and the Eigg one to a rough copy' (Campbell 1961: 41). Tá dealramh leis an tuairim sin, dar liom, ní hamháin i gcás Bìrlinn Chlann Raghnaill (Thomson 1996: 181; Black 2012: 106). Thar rud ar bith eile, ní féidir a bheith cinnte go mbeadh Raghnall Dubh sásta an dán a roinnt le MacLathagain; bhí rún aige díolaim dá chuid féin a fhoilsiú.

Is eol do scoláirí le fada go bhfuair MacLathagain cuid de dhánta Iain MhicCodrum, alias John MacCodrum / Iain mac Fhearchair (1700-1779), timpeall na bliana 1770 (Matheson 1938: xi; Gillies 2017: 135). Le páipéir eile MhicLathagain, in GUL LS 1042/68, atá siad sin caomhnaithe anois. File mór le rá as Uibhist a Tuath a bhí i MacCodrum; anois, agus litreacha seo MhicLathagain curtha sa mheá, is féidir talamh slán a dhéanamh de go bhfuarthas na dánta sin in Uibhist. Tá scagadh déanta ag MacGill'İosa ar an rud ar a dtugann sé 'the evidence for Mac Mhaighstir Alastair's role in MacCodrum's story' (Gillies 2017: 132; Gillies 2011: 83). Bhí dlúthbhaint ag Mac Mhaighstir Alasdair le hoileáin Uibhist, go mór mór le hUibhist a Deas agus Beinn na Faoghla (Black 1986: 3-5). Is léir go raibh dlúthchaidreamh ag an dá fhile lena chéile, bíodh is nach léir i gcónaí dúinn na mionsonraí. Is i mbailiúchán MhicLathagain atá an leagan is luaithe den amhrán Smeòrach Chlann Dòmbnaill le MacCodrum a bhfuil dlúthbhaint aige le Smeòrach Cblann Raghnaill Mhic Mhaighstir Alasdair (Gillies 2017: 133; 135). Bhí MacCodrum i dteagmháil lena raibh fágtha faoin am sin de Chlann Mhuirich, an teaghlach léannta a shíolraigh ó Mhuiríoch Albanach Ó Dálaigh ( $f$. 1220idí), dream a bhí i seirbhís Chlann Raghnaill ar feadh na gcéadta bliain roimhe sin (Gillies 2017: 136). Tá neart fianaise ann go raibh teagmhálacha leanúnacha idir Alasdair Mac Mhaigh- stir Alasdair agus baill de Chlann Mhuirich in Uibhist chomh maith; go deimhin fuair Mac Mhaighstir Alasdair agus a mhac Raghnall lámhscríbhinní agus ábhar eile uathu (Black 1986: 12; Campbell 2000: 136). Tugann MacLathagain féin le fios i litreacha eile gur casadh 'the MacMhuirich' air, agus bíodh is nach sonraíonn sé cé acu turas 1770 nó turas 1774 a bhí i gceist, ba dheacair a shamhlú nach dtapódh MacLathagain an deis teagmháil le Clann Mhuirich ar chaon cheann den dá thuras go hUibhist.

Ba é a mhaígh Ruairidh MacThòmais i dtaobh na teanga sna leaganacha éagsúla de Birlinn Chlann Raghnaill "[t]hat a Uist rather than a Mainland pronunciation surfaces especially in part of the Eigg text, but also in the McL and RIA versions'. Is beag cúnamh é sin agus sinn ag iarraidh oibriú amach cén áit ar bhailigh MacLathagain an dán, áfach. Nuair a mhaíonn MacThòmais (Thomson 1996: 154, nótaí 1463 agus 1467) gur a Uist pronunciation' atá le léamh sa litriú achdair ( - acair), racbdain ( - racain) etc., mar shampla, tá an ceart aige sa mhéid is go bhfuil an fuaimniú sin le cloisteáil in Uibhist, ach tá sé ar fáil i gceantair áirithe ar an tír mór chomh maith céanna, ceantair a bhí faoi smacht Chlann Raghnaill san áireamh (Ó Maolalaigh 2010: 382). Ní gá dul i bhfad scéil anseo leis, ach, go hachomair, chaithfí scagadh córasach a dhéanamh ar theanga na leaganacha sula bhféadfaí glacadh le háitiú MhicThòmais.

Dá éis sin is uile, is leor an fhianaise a pléadh thuas, dar liom, le rá gur dócha gur in Uibhist a fuair MacLathagain a chóip de Birlinn Chlann Raghnaill i samhradh na bliana 1770 - sé bliana sular fhoilsigh Raghnall Dubh an dán i nDíolaim Eilean Eige. Am éigin i ndiaidh Feabhra 1771 a chuir MacLathagain cóip ar fáil dá chomhfhreagraí Éireannach, Cathal Ó Conchúir Bhéal Átha na gCarr.

\section{Conclúid}

Tá roinnt mhaith de luathstair théacsúil Bìrlinn Cblann Raghnaill scagtha san aiste seo agus soiléiriú dá réir tugtha ar chuid de na sonraí bunúsacha a bhaineann lena bhailiú agus lena sheachadadh. Is é Seumas MacLathagain a bhreac an chóip de Birlinn Cblann Raghnaill atá anois in Acadamh Ríoga na hÉireann. Is i samhradh na bliana 1770 a bhailigh MacLathagain an dán, ó fhoinse scríofa, le linn dó a bheith ar thuras 
thart fud Innse Gall. Is é is dóchúla gur bailíodh in Uibhist é; is cinnte gur bailíodh roinnt mhaith dánta agus amhrán eile ar an turas céanna. Fágann sé seo go raibh teacht ag Seumas MacLathagain ar chóip iomlán den dán sé bliana sular fhoilsigh mac an fhile é i nDíolaim Eilean Eige. Bíodh is nár bhailigh MacLathagain an dán iomlán go dtí 1770, is léir go raibh Bìrlinn Chlann Raghnaill ina ábhar comhfhreagrais i measc scoláirí na Gaeilge Albanaí roimhe sin, cé nach maireann an comhfhreagras seo i measc litreacha MhicLathagain, go bhfios dom. Tá rún agam scagadh a dhéanamh ar luathfhoinsí uile an dáin amach anseo; ach na leaganacha neamhiomlána a chur san áireamh, d'fhéadfaí tuilleadh fós a rá faoi stair an dáin, rud nach féidir a dhéanamh anseo cheal spáis.

Tá na himpleachtaí ar fad a pléadh san aiste seo le léamh as an bhfianaise atá caomhnaithe in Acadamh Ríoga na hÉireann, fianaise a eascraíonn as cairdeas MhicLathagain le Cathal Ó Conchúir. Níos minice ná a chéile, samhlaítear na ceangail liteartha idir Gaeil Éireann agus Alban i dtéarmaí na tréimhse Clasaicí amháin. Is lú aird atá dírithe ag scoláirí ar na ceangail a lean isteach san ochtú agus sa naoú céad déag. Ach má ba thanaí féin iad, ba iad na téada dúchais seo a thug ar dhuine de phríomhfhilí na Gaeilge lena linn, Alasdair Mac Mhaighstir Alasdair, Birlinn Chlann Raghnaill a thabhairt i dtír ag 'barraibh Charraig Fhearghais', mar a bheadh, i dtéarmaí Raghnaill MhicIlleDhuibh, 'a hand outstretched to Gaelic Ireland' ann (Black agus Carruthers 2015). Ba iad na ceangail chéanna a d'fhág go raibh súil ag foireann Albanach an tsoithigh le 'biadh is deoch gun airceas' in Éirinn, ba iad ba bhunchloch faoi chairdeas Uí Chonchúir le MacLathagain agus faoin spéis a chuir siad i gcultúr agus i litríocht a chéile. Ní beag iad.

\section{Giorrúcháin}

ARÉ: Acadamh Ríoga na hÉireann

FGB: Foclóir Gaeilge-Béarla

GUL: Glasgow University Library

\section{Nóta buíochais}

Tá mé buíoch den Ollamh Uilleam MacGill'İosa a phléigh dréacht den aiste seo liom agus de na piarmheastóirí as na moltaí úsáideacha a rinne siad.

\section{SAOTHAIR A CEADAÍODH}

Bateman, M. (2008) 'Boats in the Gaelic imagination'. In: Scottish Gaelic Studies 24: 53-72.

Bateman, M. (2012) 'The bard and the birlinn'. In: Dressler, C. agus Stiùbhart, D.U. (eag.) Alexander MacDonald: Bard of the Gaelic Enlightenment I Alasdair Mac Mhaighstir Alasdair: Bàrd an t-Soillearachaidh Ghàidhealaich. Kershader: The Islands Book Trust: 74-84.

Black, R. (1986) Mac Mhaighstir Alasdair: The Ardnamurchan Years. Inverness: The Society of West Highland \& Island Historical Research. Black, R. (2001) An Lasair: Anthology of 18th Century Scottish Gaelic Verse. Edinburgh: Birlinn.

Black, R. (2006) 'Alasdair mac Mhaighstir Alasdair and the new Gaelic poetry'. In: Manning, S., Brown, I., Clancy, T. O., Pittock, M. (eag.) The Edinburgh History of Scottish Literature: Enlightenment, Britain and Empire (1707-1918). Edinburgh: Edinburgh University Press: 110-124. Black, R. (2012) 'Solving the riddle of Fàilte do Mhòrar'. In: Dressler, C. agus Stiùbhart, D.U. (eag.) Alexander MacDonald: Bard of the Gaelic Enlightenment / Alasdair Mac Mbaighstir Alasdair: Bàrd an t-Soillearachaidh Ghàidhealaich. Kershader: The Islands Book Trust: 104115. Black, R. agus Carruthers, G. (2015) 'The eighteenth century'. In: Sassi, C. (eag.) The International Companion to Scottish Poetry. Glasgow:

Association for Scottish Literary Studies: 54-63. Campbell, J.L. (1961) 'The Royal Irish Academy text of Birlinn Chlann Raghnaill. In: Scottish Gaelic Studies 9.1: 39-79.

Campbell, J.L. (1984 [1933]) Highland Songs of the Forty-Five. Edinburgh: Scottish Gaelic Texts Society.

Campbell, J.L. (2000) 'Ranald MacDonald and the Eigg Collection'. In: Cheape, H. (eag), A Very Civil People: Hebridean Folk, History and Tradition. Edinburgh: Birlinn: 134-137.

Dressler, C. agus Stiùbhart, D.U. (eag.) (2012) Alexander MacDonald: Bard of the Gaelic Enlightenment / Alasdair Mac Mbaighstir Alasdair: Bàrd an t-Soillearachaidh Ghàidhealaich. Kershader: The Islands Book Trust. 
Dymock, E. (2009) 'Interpreting the symbol of the Cuillin: The relationship between Sorley MacLean's 'An Cuilithionn' and his 'Cuillin Praise Poem'. In: Scottish Gaelic Studies 25: 143-180.

Dziennik, M. (2015) The Fatal Land: War, Empire, and the Highland Soldier in British America. New Haven: Yale University Press.

Gibbons, L. agus O'Conor, K. (eag.) (2015) Charles O'Conor of Ballinagare, 1710-1791: Life and Works. Dublin: Four Courts Press.

Gillies, W. (2011) 'Some eighteenth-century developments in Scottish Gaelic poetry'. In: Ahlqvist, A. agus O'Neill, P. (eag.) Language and Power in the Celtic World: Papers from the Seventh Australian Conference of Celtic Studies. Sydney: Celtic Studies Foundation, The University of Sydney: 61-95.

Gillies, W. (2012) 'Reflections on Alexander MacDonald and Canna'. In: Dressler, C. agus Stiùbhart, D.U. (eag.) Alexander MacDonald: Bard of the Gaelic Enlightenment / Alasdair Mac Mhaighstir Alasdair: Bàrd an t-Soillearachaidh Ghàidhealaich. Kershader: The Islands Book Trust: 115-122.

Gillies, W. (2013a) 'A well of wine in her stern'. In: O'Neill, P. (eag.) The Land beneath the Sea: Essays in Honour of Anders Ahlqvist's Contribution to Celtic Studies in Australia. Sydney: Celtic Studies Foundation, The University of Sydney: 75-85. Gillies, W. (2013b) 'The Book of the Dean of Lismore: The literary perspective'. In McClure, J.D. agus Williams, J.H. (eag.) Fresche Fontanis: Studies in the Culture of Medieval and Early Modern Scotland. Newcastle: Cambridge Scholars Publishing: 179-216.

Gillies, W. (2017) “The Mavis of Clan Donald”: Engaging with John MacCodrum'. In: Ahlqvist, A. agus O'Neill, P. (eag.) Germano-Celtica: $A$ Festschrift for Brian Taylor. Sydney: Celtic Studies Foundation, The University of Sydney: 123-151. Gillies, W. (2020) 'Iceberg tip or floating island? The Harlaw Brosnachadh revisited'. In: Proceedings of the Harvard Celtic Colloquium 38: 1-46.

Innes, S. (2016) 'Fionn and Ailbhe's riddles between Ireland and Scotland'. In: Boyd, M. (eag.) Ollam: Studies in Gaelic and Related Traditions in Honour of Tomás Ó Cathasaigh. Madison NJ: Dickinson

University Press: 271-285.

MacDonald, C. (1996) Moidart: Among the

Clanranalds. J. Watt (eag.). Edinburgh: Birlinn.

MacGill-Eain, S. (1985) Ris a' Bhruthaich: The Criticism and Prose Writings of Sorley MacLean. W. Gillies (eag.). Stornoway: Acair.

MacLeod, A. (1933) Sàr Òrain: Three Gaelic Poems. Glasgow: An Comunn Gàidhealach.

Martin, M. (1716) A Description of the Western Islands of Scotland. London.

Matheson, W. (1938) The Songs of John MacCodrum. Edinburgh: Scottish Gaelic Texts Society.

Meek, D. (1999) Léirmheas ar Thomson (1996). In: Scottish Gaelic Studies 19: 258-261.

Murphy, G. agus Wulff, W. (1936) Catalogue of Irish Manuscripts in the Royal Irish Academy: Fasciculus xviii. Dublin: Royal Irish Academy.

Ó Catháin, D. (2015) 'Some account of Charles O'Conor and literacy in Irish in his time'. In: Gibbons, L. agus O'Conor, K. (eag.) Charles O'Conor of Ballinagare, 1710-1791: Life and Works. Dublin: Four Courts Press: 28-51.

O'Conor, C. (1775) (eag.) The Ogygia vindicated, against the objections of Sir George Mac Kenzie. Dublin: Faulkner.

O'Conor, C. (1818) Bibliotheca MS Stowenensis: A Descriptive Catalogue of the Manuscripts in the Stowe Library. Buckingham: Seeley.

Ó Maolalaigh, R. (2010) 'The sound of silence: Some structural observations on preaspiration in Scottish Gaelic'. In: McLeod, W., Burnyeat, A., Stiùbhart, D.U., Clancy, T.O., agus Ó Maolalaigh, R. (eag.) Bile ós Chrannaibh: A Festschrift for William Gillies. Ceann Drochaid: Clann Tuirc: 365-404.

Ó Muircheartaigh, P. (2016) 'Fin as Ossian revisited: A Manx ballad in Belanagare and its significance'. In: Zeitschrift für celtische Philologie 63: 95-127.

Ó Muirthile, L. (2015) Aois Fir. Baile Átha Cliath: Cois Life.

Parsons, G. (2013) 'Gaelic bards and Norwegian rigs'. In: Journal of the North Atlantic 4: 26-34.

Pittock, M. (2012) 'Jacobite society and culture in the age of Alasdair mac Mhaighstir Alasdair'. In: Dressler, C. agus Stiùbhart, D.U. (eag.) Alexander MacDonald: Bard of the Gaelic Enlightenment 
I Alasdair Mac Mhaighstir Alasdair: Bàrd an t-Soillearachaidh Ghàidhealaich. Kershader: The Islands Book Trust: 56-62.

Savonius-Wroth, C. (2019) 'Bardic ministers:

Scotland's Gaelic-speaking clergy in the Ossian controversy'. In: Eighteenth-Century Studies 52.2: 225-243.

Stiùbhart, D.U. (2012) 'Alexander MacDonald, catechist and schoolteacher'. In: Dressler, C. agus Stiùbhart, D.U. (eag.) Alexander MacDonald: Bard of the Gaelic Enlightenment / Alasdair Mac Mhaighstir Alasdair: Bàrd an t-Soillearachaidh Ghàidhealaich. Kershader: The Islands Book Trust: 13-24.

Thomson, D.S. (1990) An Introduction to Gaelic Poetry. Edinburgh: Edinburgh University Press. Thomson, D.S. (eag.) (1992) The McDiarmid MS Anthology: Poems and Songs Mainly Anonymous from the Collection Dated 1770. Edinburgh: Scottish Gaelic Texts Society. Thomson, D.S. (1994) 'The McLagan MSS in Glasgow University Library: A survey'. In: Transactions of the Gaelic Society of Inverness 58: 406-442.

Thomson, D.S. (eag.) (1996) Alasdair Mac Mhaighstir Alasdair: Selected Poems. Edinburgh: Scottish Gaelic Texts Society.

Turner, A. (2018) “A Tour to the Ebudae" by Rev. James MacLagan, 1774'. In: Transactions of the Galeic Society of Inverness 68: 237-260.

Ward, R.E., Wrynn, J.F. agus Coogan Ward, C. (eag.) (1988) Letters of Charles O'Conor of Belanagare: A Catholic Voice in Eighteenth-Century Ireland. Washington: Catholic University of America. 